\title{
Automated EJB2 to EJB3 Migration
}

\author{
Isha Mittal, \\ AMITY University \\ Noida, India
}

\author{
J.S. Sodhi, PhD \\ AMITY University \\ Noida, India
}

\begin{abstract}
Conversion from some old technology to new technology is requirement for today's world, our IT industry was started growing up in late 90 's. At that time there were lots of development comes in different industry, banking system had changed drastically, it's come online with online solutions, ATM takes place for withdrawing money from the bank, similarly manufacturing industry grows with IT etc.

We had used advance technology of that time for providing solution to the respective industry. As the time changes there are some advancement in each field so IT has also comes with lots of new technology \& configurations, so that we can solve the real world problem more accurately with cheap prize.

We are providing a automate migration tool that will migrate EJB2.1 (old) to EJB3.0 (new)[1], EJB2.1 have several disadvantages like it's do not have annotation where annotations are server independent configurations.
\end{abstract}

\section{Keywords}

EJB2, EJB3, migration, business layer, Ejb2 to Ejb3 migration, time saving,

\section{INTRODUCTION}

Develop a tool to automate the generation of EJJB3.0 entity (with JPA) and corresponding DAO objects using available EJB2.1 artifacts such as XDoclet bean and the code (EJB2.1 files, Weblogic AS specific deployment descriptors, Data Transfer Objects (DTOs)) generated using it.[2]

The artifacts generated by the tool should follow the listed standards.

\section{- $\quad$ EJB3.0 Entity}

- Should list the files that were used to generate the entity in class level comments.

- Should contain standard JPA annotations like “@Entity", “@Table”, “@NamedQueries”, “@TransactionAttribute”, “@Column”, “@Id”, “@SequenceGenerator”, “@GeneratedValue”, and “@Lob” wherever applicable.

- Should list the EJB2.1 deployment descriptor annotations that are not processed by the tool as "TODO" in class level comments

- Should extend CLBaseEntity<PK Type $>$ and implement "getCompareables()" and "getPrimaryKey()" methods.

- In case of composite primary key, a separate primary key class should be generated annotated with “@Embeddable” annotation.
- In case of composite primary key, entity should have an instance variable of the primary key class with “@EmbeddedId" annotation.

- $\quad$ DAO Interface

- Should extend CLBaseDao<EJB3.0-Entity, PK Type>

- Should have declarations of all finder methods.

- Should have "TODO" comment that list all the custom methods (not including getters/setters of entity fields and "getData()" methods) with parameters.

- DAO Implementation Class

$\circ \quad$ Should extend CLBaseDaoImpl< EJB3.0-Entity, PK Type> and implement <EJB3.0-Entity>Dao

- Should have implementations of all finder methods.

○ Should have "TODO" comment that list all the custom methods (not include getters/setters of entity fields and "getData()" methods) with parameters

\subsection{Analysis}

\subsubsection{Identification of Data}

In this analysis all the data that would represent an entity attributes was recognized. The following is the list of such attributes.

- $\quad$ Entity name and fields

- Table name and columns

- $\quad$ Finder methods and queries

- $\quad$ Entity relationships

\subsubsection{Identification of Artefacts}

In this analysis all the artifacts that would be used to aggregate entity attributes was recognized. The following is the list of such artifacts.

- $\quad$ ejb-jar.xml

- weblogic-cmp-rdbms-jar.xml

- $\quad$ weblogic-ejb-jar.xml

- Local interface corresponding to each entity bean

- Data Transfer Objects corresponding to each entity 


\section{Patterns recognized}

- In-case of deployment descriptors each module contains these xml files in <path>|target|classes|META-INF|

- The corresponding jar file contain the DTOs and local interfaces of the entity beans are accessible from <path> >targetl

\subsubsection{Identification of Development Methodologies}

In this analysis development methodologies were identified that would be used to generate EJB3.0 and DAO code. The following is the list of such artifacts.

- $\quad$ XML Transformation using JAXP

- $\quad$ Saxon Processor (XSLT 2.0 compatible)

- $\quad$ Logging using $\log 4 \mathrm{j}$

- Directory scanning based on pattern

\subsection{Design}

The tool consists of 2 mains components as depicted in Figure1

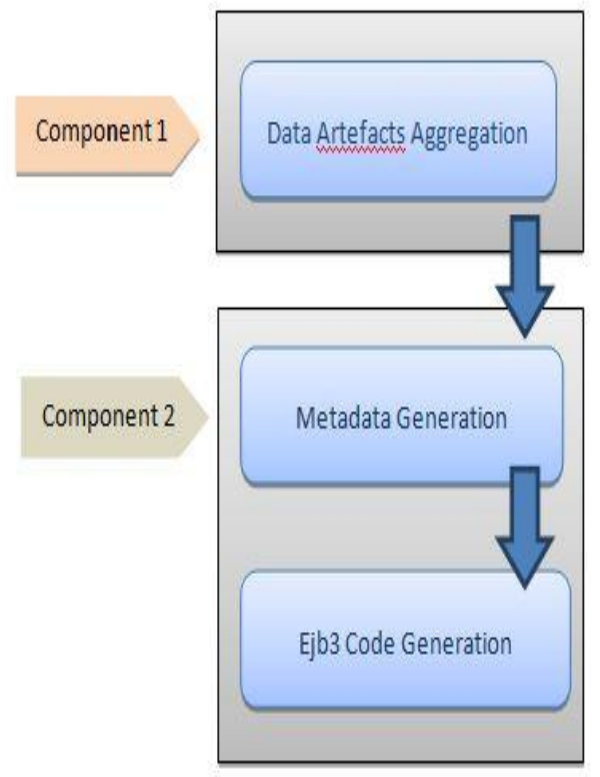

\subsubsection{Data Artefacts Aggregation}

This component searches a particular directory for certain set of files by making use of Regular Expression and generates 2 files. Figure 2 depicts this process.

The file patterns used to scan the required directory are

- $\quad$ Pattern used to filter 3 types of files - "(^^weblogiccmp-rdbms-jar.xml)|(^ejb-jar.xml)|(^ weblogic-ejb-jar.xm)"

- Pattern used to make sure that files come from certain type of directory only - ".*classes IIIIMETA-INF.*"
- $\quad$ Pattern used to filter jar file that contains DTOs and local interfaces - "IDD.*-SNAPSHOT.jar\$"

The file crawler generates two files after scanning the depths of the required directory.

- $\quad$ An xml file (filteredDDPaths.xml) that contains the absolute paths of the deployment descriptor files for each module.

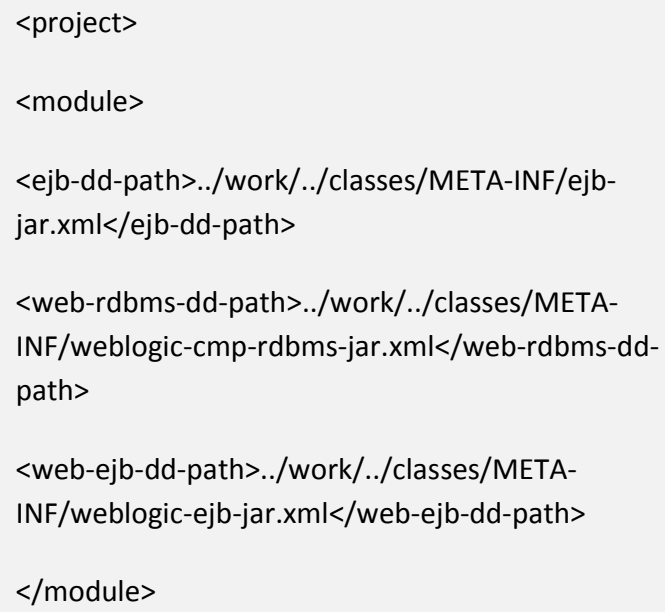

- A file (jarPaths.txt) that lists the absolute paths of the jar files that contain DTOs and local interfaces for each module. This list would be used to set Java "classpath" and is a pre-requisite for "Data Transformation"

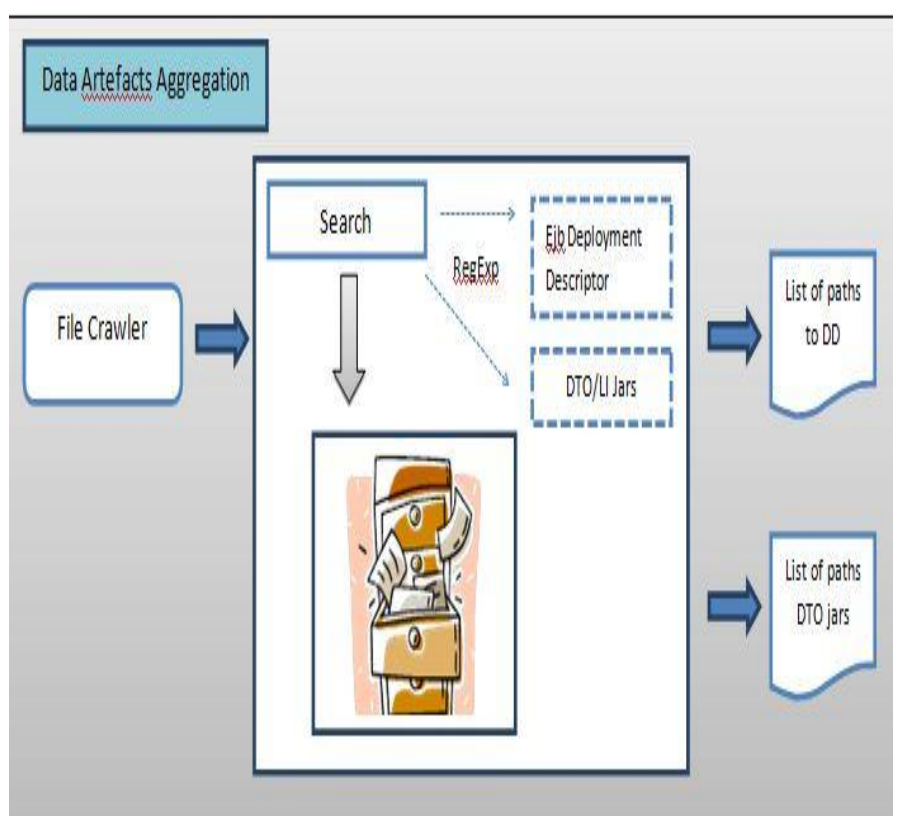

Figure 2-Data Artifacts Aggregation 


\subsubsection{Data Transform}

The data transformation component provides the functionality of meta-data generation and finally EJB3.0/DAO code generation. The following Figure 3 depicts this process.

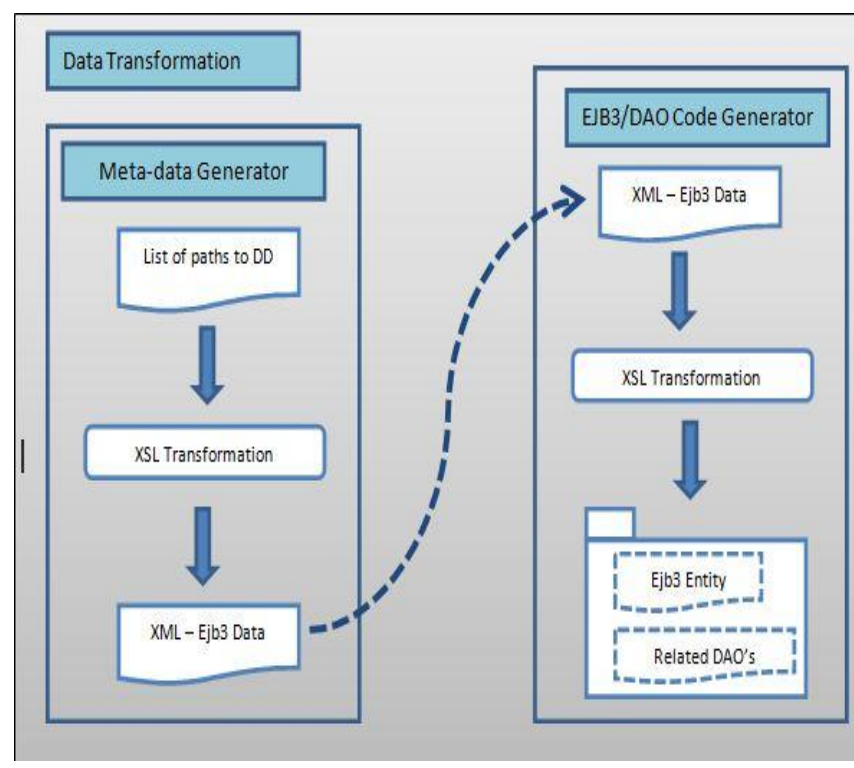

Figure 3-Data Transformation

\subsubsection{Meta-data Generation}

This component gathers all the information segregated in different files for each entity and place it one xml file (entitydata. $x \mathrm{ml}$ ). XSL transformation would be incorporated to work with different $\mathrm{xml}$ files and access DTOs present in jar files to aggregate the data. For each "module" element in "filteredDDPaths.xml", a separate "entity-data.xml" file would be generated.

\subsubsection{EJB3.0/DAO Code Generation}

This component uses the meta-data file "entity-data.xml" and performs an XSL transformation to generate the Java code. The XSL transformation will generate EJB3.0 entity and it corresponding DAO objects referencing the generic DAO.

\section{IMPLEMENTATION}

\subsection{Development Modules}

Eclipse IDE is used for tool development.

\subsubsection{3rdParty}

- Apache

○ $\quad \log 4 \mathbf{j}-1.2 .16 . j a r-$ Application logging

- Saxon-9.1B

○ $\quad$ saxon9-dom.jar - XML document parsing

- $\quad$ saxon9.jar - XSLT processor

- J2EE

j2ee-1.4.jar - Required because the tool loads EJB2.1 files (local interfaces) using reflection for scanning

\subsubsection{Common}

\subsubsection{Utilities}

- ConfigReader.java - This class is used to load base configuration file.

- $\quad$ ToolResourceBundle.java - This class maintains resource bundle for given file.

- Constants.java - This interface holds application constants values.

- $\quad$ ClassAnalyzer.java - This component loads a class dynamically and retrieves its properties.

- DataInterpreter.java - This class is to analyse the data for blank or empty values.

- $\quad$ FileUtility.java - This class provides various file I/O functionality.

\subsubsection{Resources}

- $\quad$ ejb2-to-ejb3-config.properties Tool configuration file

- $\quad \log 4 \mathbf{j}$.properties - Tool $\log 4 \mathrm{j}$ configuration file

- CatalogManager.properties - This file tells the resolver where to look for catalog files and sets configuration options.

- $\quad$ Xml-catalog.xml - A catalog in XML provides a mapping from generic addresses to specific local directories on a given machine. A catalog can be used to locate the DTD, system entity files, and stylesheet files during processing.

- $\quad$ ejb-jar_2_0.dtd - DTD used by "ejb-jar.xml”

- $\quad$ gcode-constants.xml - This XML file holds constants that used by XSLT files.

\subsubsection{EJB2 to EJB3}

\subsubsection{Data Artefacts Aggregation}

- $\quad$ FileCrawler.java - This component searches a particular directory for certain set of files by making use of Regular Expression and generates 2 files. The first is an XML file that contains the absolute paths of the deployment descriptor files for each module. The second is a text file that lists the absolute paths of the jar files that contain DTOs and local interfaces for each module.

\subsubsection{Data Transformation}

- Meta-data Generator

○ MetadataGenerator.java - This class gathers all the information segregated in different files for each entity and place it one $\mathrm{xml}$ file (entity-data-i.xml). XSL transformation would be incorporated to work with different $\mathrm{xml}$ files and access DTOs present in jar files to aggregate the data. For each "module" element in "filteredDDPaths.xml", a separate "entity-data-i.xml" file would be generated.

- meta-data-builder.xsl - XSLT file used for metadata generation

\section{- $\quad$ EJB3.0/DAO Code Generator}

- CodeGenerator.java - This class uses the metadata file "entity-data-i.xml" and performs an XSL 
transformation to generate the Java code. The XSL transformation will generate EJB3.0 entity and it corresponding DAO objects referencing the Generic DAO.

○ code-generator.xsl - XSLT file used for EJB3.0/DAO generation.

\subsubsection{Build}

\subsubsection{Ant Build Process}

The build process will package the common and EJB2 to EJB3 java code in separate jar files. The resources would not be packaged within the jar file. This would provide flexibility to the user to modify configuration settings without worrying about executing the build again. I

- $\quad$ ejb2-to-ejb3-tool-build.xml

- $\quad$ ejb2-to-ejb3-tool-build.properties

\subsection{Execution Process}

The EJB2 to EJB3 migration tool would be executed using a batch process (ejb2_to_ejb3_tool.bat). The following would be the various stages.

- Data artefacts aggregation

- $\quad$ Set java classpath using the jar file paths set in "jarPaths.txt

- Data transformation

\section{THINGS TO REMEMBER}

The following should be considered while integrating the generated code.

1. I has been noticed that in the EJB2.1 code, named queries use reference to the entity attribute name that do not match the actual declared variable. Wherever such instances are encountered, rectify the named query.

2. If a "TODO" related to "The following table column is mapped to an entity field as well as to an entity relationship attribute" exists; however the related attributes are defined in separate classes (e.g. Entity and PK class), ignore and remove this TODO.

3. After applying fixes for a TODO, it should be removed from the code.

4. If an entity exists more than once in the EJB jar, it should be distinguished by custom entity name by setting the "name” attribute of “@Entity” annotation.

\subsection{Tool}

\subsection{Structure}

The tool deliverable is in form of a zip archive file "ejb2-toejb3-migration.zip". Its structure is as follows.

\section{ejb2-to-ejb3-migration.zip}

formatter

tool

lib

resources

xslt-files

$\$$ ejb2-to-ejb3-tool.jar

i: ejb2_to_ejb3_tool.bat

\begin{tabular}{|l|l|}
\hline Directory/File & Description \\
\hline formatter & $\begin{array}{l}\text { Contains Eclipse IDE } \\
\text { code formatting } \\
\text { preferences file. }\end{array}$ \\
\hline Tool & $\begin{array}{l}\text { Base folder for } \\
\text { migration tool. }\end{array}$ \\
\hline Lib & $\begin{array}{l}\text { Contains 3d party and } \\
\text { common project jar } \\
\text { files. }\end{array}$ \\
\hline resources & $\begin{array}{l}\text { Contains } \\
\text { configuration files. }\end{array}$ \\
\hline xsit-files & $\begin{array}{l}\text { Contains XSL } \\
\text { transformation file. }\end{array}$ \\
\hline ejb2_to_ejb3_tool.bat & $\begin{array}{l}\text { Batch file to execute } \\
\text { the tool. }\end{array}$ \\
\hline ejb2-to-ejb3-tool.jar & Migration tool jar. \\
\hline
\end{tabular}

\subsection{Configuration}

The tool can be configured by using "ejb2_to_ejb3\Utilityไresourceslejb2-to-ejb3config.properties" file. It lists different keys that can be set as per project requirements.

\begin{tabular}{|l|l|l|}
\hline $\begin{array}{l}\text { S. } \\
\text { No }\end{array}$ & Key & Purpose \\
\hline 1. & $\begin{array}{l}\text { data.lookup.dir } \\
\text { path }\end{array}$ & $\begin{array}{l}\text { Directory path for } \\
\text { searching } \\
\text { artifacts. } \\
\text { the } \\
\text { e.g.//ip.address/c\$/work } \\
\text { /flood" }\end{array}$ \\
\hline 2. & $\begin{array}{l}\text { data.artifacts.di } \\
\text { r.name }\end{array}$ & $\begin{array}{l}\text { Directory for listing } \\
\text { the filtered artifacts. } \\
\text { e.g. } \\
\text { "//ip.address/c\$/ejb2 } \\
\text { to_ejb3/artifacts-list- }\end{array}$ \\
\hline
\end{tabular}




\begin{tabular}{|c|c|c|}
\hline & & files" \\
\hline 3. & $\begin{array}{l}\text { data.artifacts.d } \\
\text { d.paths }\end{array}$ & $\begin{array}{l}\text { Name of the file that } \\
\text { lists the path to } \\
\text { filtered files. } \\
\text { e.g. } \\
\text { "filteredDDPaths.xml } \\
\text { ", }\end{array}$ \\
\hline 4. & $\begin{array}{l}\text { data.artifacts.ja } \\
\text { r.paths }\end{array}$ & $\begin{array}{l}\text { Name of the file that } \\
\text { lists the jar file names } \\
\text { that are to be set in } \\
\text { Java classpath. These } \\
\text { jar files contain } \\
\text { DTO's and Local } \\
\text { interfaces that would } \\
\text { be interpreted for } \\
\text { EJB3 entity } \\
\text { properties. } \\
\text { e.g. "jarPaths.txt" }\end{array}$ \\
\hline 5. & $\begin{array}{l}\text { data.meta.dir.n } \\
\text { ame }\end{array}$ & $\begin{array}{l}\text { Directory path of the } \\
\text { generated meta-data } \\
\text { files } \\
\text { e.g. } \\
\text { "//ip.address/c\$/ejb2_- } \\
\text { to_ejb3/meta-data- } \\
\text { files" }\end{array}$ \\
\hline 6. & $\begin{array}{l}\text { meta.data.file.n } \\
\text { ame }\end{array}$ & $\begin{array}{l}\text { Name of the meta- } \\
\text { data file } \\
\text { e.g. "entity-data-" }\end{array}$ \\
\hline 7. & $\begin{array}{l}\text { meta.data.file.e } \\
\text { xtension }\end{array}$ & $\begin{array}{l}\text { File extension of } \\
\text { meta-data file } \\
\text { e.g. ".xml" }\end{array}$ \\
\hline 8. & $\begin{array}{l}\text { filtered.data.fil } \\
\text { e.root }\end{array}$ & $\begin{array}{l}\text { Artifacts xml file root } \\
\text { node name } \\
\text { e.g. "project" }\end{array}$ \\
\hline 9. & $\begin{array}{l}\text { filtered.data.fil } \\
\text { e.element.mod } \\
\text { ule }\end{array}$ & $\begin{array}{l}\text { Artifacts } \mathrm{xml} \text { file } \\
\text { module node name } \\
\text { e.g. "module" }\end{array}$ \\
\hline 10. & $\begin{array}{l}\text { filtered.file.ele } \\
\text { ment.ejb.dd.pat } \\
\text { h }\end{array}$ & $\begin{array}{l}\text { Artifacts } \\
\text { jar.xml" } \text { "ejb- } \\
\text { module child node } \\
\text { name } \\
\text { e.g. "ejb-dd-path" }\end{array}$ \\
\hline
\end{tabular}

\begin{tabular}{|c|c|c|}
\hline 11. & $\begin{array}{l}\text { filtered.file.ele } \\
\text { ment.web.rdbm } \\
\text { s.dd.path }\end{array}$ & $\begin{array}{l}\text { Artifacts "weblogic- } \\
\text { cmp-rdbms-jar.xml" } \\
\text { xml file module child } \\
\text { node name } \\
\text { e.g. "web-rdbms-dd- } \\
\text { path" }\end{array}$ \\
\hline 12. & $\begin{array}{l}\text { filtered.file.ele } \\
\text { ment.web.ejb.d } \\
\text { d.path }\end{array}$ & $\begin{array}{l}\text { Artifacts "weblogic- } \\
\text { ejb-jar.xml" xml file } \\
\text { module child node } \\
\text { name } \\
\text { e.g. "web-ejb-dd- } \\
\text { path" }\end{array}$ \\
\hline 13. & $\begin{array}{l}\text { data.generated. } \\
\text { code.dir.option }\end{array}$ & $\begin{array}{l}\text { Flag to switch } \\
\text { between custom } \\
\text { output directory } \\
\text { location. Valid values } \\
\text { are "CUSTOM" and } \\
\text { "LOOK_UP_DIR" } \\
\text { e.g. "CUSTOM" }\end{array}$ \\
\hline 14. & $\begin{array}{l}\text { data.generated. } \\
\text { code.custom.di } \\
\text { r.name }\end{array}$ & $\begin{array}{l}\text { Directory location } \\
\text { where the code is } \\
\text { generated. Applicable } \\
\text { if the directory option } \\
\text { is "CUSTOM" } \\
\text { e.g. } \\
\text { "//ip.address/c\$/ejb2_ } \\
\text { to_ejb3/output" }\end{array}$ \\
\hline 15. & $\begin{array}{l}\text { data.generated. } \\
\text { code.sub.src.m } \\
\text { ain }\end{array}$ & $\begin{array}{l}\text { Sub-directory name } \\
\text { where the EJB3 code } \\
\text { generated by the tool } \\
\text { would be placed } \\
\text { e.g. "src/main/java/" }\end{array}$ \\
\hline 16. & $\begin{array}{l}\text { data.generated. } \\
\text { code.sub.src.te } \\
\text { st }\end{array}$ & $\begin{array}{l}\text { Sub-directory name } \\
\text { where the EJB3 test } \\
\text { code generated by the } \\
\text { tool would be placed } \\
\text { e.g. "src/test/java/" }\end{array}$ \\
\hline 16. & $\begin{array}{l}\text { dd.file.name.pa } \\
\text { ttern }\end{array}$ & $\begin{array}{l}\text { Pattern of the files to } \\
\text { be filtered by the file } \\
\text { crawler } \\
\text { e.g. " }\left({ }^{\wedge} \text { weblogic- }\right. \\
\text { cmp-rdbms- } \\
\text { jar.xml }) \mid\left(\wedge^{\wedge} \text { ejb- }\right. \\
\text { jar.xml } \mid\left(\wedge^{\wedge} \text { weblogic- }\right.\end{array}$ \\
\hline
\end{tabular}




\begin{tabular}{|c|c|c|}
\hline & & ejb-jar.xml)" \\
\hline 17. & $\begin{array}{l}\text { dd.file.absolute } \\
\text {.path.pattern }\end{array}$ & $\begin{array}{l}\text { Filtered deployment } \\
\text { descriptor files } \\
\text { absolute path pattern } \\
\text { e.g. } \\
\text { ".*classes IIIIMETA- } \\
\text { INF.*" }\end{array}$ \\
\hline 18. & $\begin{array}{l}\text { jar.file.path.filt } \\
\text { er }\end{array}$ & $\begin{array}{l}\text { Filter for jar file } \\
\text { location } \\
\text { e.g. "classes" }\end{array}$ \\
\hline & src.dir.filter & $\begin{array}{l}\text { Filter for source } \\
\text { directory location } \\
\text { path } \\
\text { e.g. "target/classes" }\end{array}$ \\
\hline 19. & $\begin{array}{l}\text { entity.dao.pack } \\
\text { age.filter.val }\end{array}$ & $\begin{array}{l}\text { Filter for establishing } \\
\text { the package for } \\
\text { entities and daos } \\
\text { e.g. "interfaces." }\end{array}$ \\
\hline 20. & $\begin{array}{l}\text { jar.file.name.pa } \\
\text { ttern }\end{array}$ & $\begin{array}{l}\text { Pattern of the jar file } \\
\text { to be filtered } \\
\text { e.g. } \\
\text { SNAPSHOT.jar\$" }\end{array}$ \\
\hline 21. & $\begin{array}{l}\text { meta.data.build } \\
\text { er }\end{array}$ & $\begin{array}{l}\text { Location of the } \\
\text { transformation file } \\
\text { for entity meta-data } \\
\text { builder } \\
\text { e.g. } \\
\text { "//ip.address/c\$/ejb2_- } \\
\text { to_ejb3/utility/xslt- } \\
\text { files/meta-data- } \\
\text { builder.xsl" }\end{array}$ \\
\hline 22. & code.generator & $\begin{array}{l}\text { Location of the } \\
\text { transformation file } \\
\text { for entity code } \\
\text { generator } \\
\text { e.g. } \\
\text { "//ip.address/c\$/ejb2_- } \\
\text { to_ejb3/utility/xslt- } \\
\text { files/code- } \\
\text { generator.xsl" }\end{array}$ \\
\hline 23. & $\begin{array}{l}\text { code.constants. } \\
\text { file }\end{array}$ & $\begin{array}{l}\text { Location of the xml } \\
\text { file that holds } \\
\text { constants used in }\end{array}$ \\
\hline
\end{tabular}

\begin{tabular}{|l|l|l|}
\hline & & $\begin{array}{l}\text { code generation } \\
\text { e.g. } \\
\text { “//ip.address/c\$/ejb2- } \\
\text { to-ejb3- } \\
\text { migration/tool/resour } \\
\text { ces/xsl- } \\
\text { constants.xml" }\end{array}$ \\
\hline 24. & $\begin{array}{l}\text { xml.transform.f } \\
\text { actory }\end{array}$ & $\begin{array}{l}\text { XML Transform } \\
\text { Factory } \\
\text { e.g. } \\
\text { "javax.xml.transform. } \\
\text { TransformerFactory" }\end{array}$ \\
\hline 25. & $\begin{array}{l}\text { Saxon.xml.tran } \\
\text { sform.factory }\end{array}$ & $\begin{array}{l}\text { XML Transform } \\
\text { Factory } \\
\text { Implementation } \\
\text { e.g. } \\
\text { "net.sf.saxon.Transfo } \\
\text { rmerFactoryImpl" }\end{array}$ \\
\hline
\end{tabular}

\subsection{Execution}

The tool can the executed by running the "ejb2_to_ejb3_tool.bat". This batch process involves 5 different stages that are listed below.

\begin{tabular}{|l|l|}
\hline S.No. & Section \\
\hline 1. & Data artifacts aggregation \\
\hline 2. & Set Java classpath \\
\hline 3. & Meta-data generation \\
\hline 4. & Code generation \\
\hline 5. & $\begin{array}{l}\text { Generated code indentation using } \\
\text { Eclipse IDE. } \\
\text { Note: The user would need to modify } \\
\text { the script for section 5 of the batch file } \\
\text { as per the local machine. }\end{array}$ \\
\hline
\end{tabular}

\section{CONCLUSION}

This paper gives an intelligent solution for migration of old ejb version ejb 2.1 to new ejb version 3.0. It can be used in the IT industry, especially for those companies which are working on migration domain. Ejb contains the business logic of our code that needs more security \& flexibility from other code; hence we can say it's a useful tool for migration your code to new code.

\section{REFERENCES}

[1] Oracle Ejb Migration 2005

[2] EJB components Migration Service and Automatic Deployment - HA 